\title{
STREAMLINES AND DETACHED WAKES IN STEADY FLOW PAST A SPHERICAL LIQUID DROP
}

\author{
G.M. Moremedi ${ }^{1}$ and D.P. Mason ${ }^{2}$ \\ ${ }^{1}$ Department of Mathematical Sciences, University of South Africa, \\ PO Box 392, Pretoria, South Africa. \\ ${ }^{2}$ Centre for Differential Equations, Continuum Mechanics and Applications \\ and School of Computational and Applied Mathematics, University of the \\ Witwatersrand, Johannesburg, Private Bag 3, Wits 2050, South Africa \\ Moremgm@unisa.ac.za, David.Mason@wits.ac.za
}

\begin{abstract}
The flow interior and exterior to a viscous liquid drop in steady motion in an unbounded quiescent fluid is investigated using the perturbation solution of Taylor and Acrivos (1964) to first order in the Reynolds number. New analytical results are derived for the detached wake behind the drop. It is found that as the viscosity of the drop tends to infinity the wake becomes attached to the surface of the drop and the results of Proudman and Pearson (1957) for a solid sphere are rederived.
\end{abstract}

Keywords- Singular perturbation solution, streamlines, boundary of standing eddies, stagnation point.

\section{INTRODUCTION}

The objective of the paper is to derive new analytical results for the streamlines and for the wake in the steady flow of a viscous fluid past a spherical liquid drop.

Van Dyke [1] applied the singular perturbation solution of Proudman and Pearson [2] for slow viscous flow past a solid sphere to analyse the attached wake behind the sphere. He found that although the perturbation solution was derived for Reynolds numbers $R e<1$ the results obtained were in good agreement with experimental and numerical results for values up to $R e=60$. The prediction of Proudman and Pearson [2] that standing eddies first appear behind the sphere at $R e=8$ agrees well with the numerical value of 8.5 obtained by Jenson [3] using the full NavierStokes equation and with the experimental value of 12 obtained by Taneda [4]. Van Dyke also found good agreement, up to $R e=60$, between the perturbation solution to first order in $R e$ and the experimental and numerical values for the length of the attached wake. For $R e \gtrsim 60$ the flow behind the sphere becomes unsteady.

We will derive new analytical results for the axisymmetric flow past a viscous liquid drop with constant surface tension using the singular perturbation solution of Taylor and Acrivos [5]. We will assume that the inter-facial tension is large so that the Weber number is small and therefore the deformation of the spherical drop is small. The perturbation solution depends on two parameters, the Reynolds number Re and the ratio of the viscosity of the drop to the viscosity of the surrounding fluid, $\kappa$. Although the perturbation solution was derived for $R e<1$, we will consider $R e>1$ as was done with the perturbation solution of Proudman and Pearson for flow past a solid sphere. There is evidence that the predictions of the perturbation 
solution of Taylor and Acrivos may be applicable for $R e>1$. For instance Wellek et al [6] found that the Taylor and Acrivos solution quite accurately predicted drop eccentricities for drop Reynolds numbers up to $R e=20$. The perturbation expansions for large $\kappa$ should be applicable to flow past a very viscous drop.

There have been several numerical investigations of flow past a liquid drop [7-10].

\section{STREAM FUNCTIONS}

The singular perturbation solution of Taylor and Activos [5] describes the steady axisymmetric motion under gravity of a viscous drop slightly deformed from the spherical shape in an unbounded quiescent fluid. The fluids are incompressible, immiscible and the interfacial tension $\sigma$ between the viscous drop and the surrounding fluid is uniform. Physical variables inside the drop are distinguished from corresponding variables outside the drop by a circumflex. A fixed spherical polar coordinate system $(r, \theta, \phi)$ is used with origin at the centre of mass of the viscous drop. All the fluid dynamical variables are dimensionless and independent of $\phi$. The characteristic length is the radius, $a$, of the spherical drop with the same volume, and the characteristic velocity is the terminal velocity $U$ of the drop. The Reynolds number, defined in terms of the parameters of the exterior fluid, and the viscosity ratio $\kappa$ are

$$
R e=\frac{U a}{\nu}, \kappa=\frac{\hat{\eta}}{\eta}
$$

where $\eta$ is the shear viscosity and $\nu=\eta / \rho$.

Taylor and Acrivos used the method of matched asymptotic expansions for the solution exterior to the drop. The straightforward expansion in powers of $R e$ is the inner expansion exterior to the drop. The inner expansion is used to analyse the exterior flow close to the drop which includes the attached wake. It was found that there is no deformation of the drop at zero order in $R e$. The boundary conditions for the first order solution are therefore imposed at $r=1$. The inner expansion exterior to the drop and to first order in $R e$ can be written as

$$
\begin{aligned}
\psi(r, \theta)= & \frac{1}{4}(r-1) \sin ^{2} \theta\left[( 1 + \frac { 1 } { 8 } ( \frac { 2 + 3 \kappa } { 1 + \kappa } ) R e ) \left(2 r-\frac{\kappa}{1+\kappa}\right.\right. \\
& \left.-\frac{\kappa}{1+\kappa} \frac{1}{r}\right)-\frac{R e}{8}\left(\frac{2+3 \kappa}{1+\kappa}\right)\left(2 r-\frac{\kappa}{1+\kappa}-\frac{\kappa}{5(1+\kappa)^{2}} \frac{1}{r}\right. \\
& \left.\left.-\frac{\kappa(6+5 \kappa)}{5(1+\kappa)^{2}} \frac{1}{r^{2}}\right) \cos \theta\right]+\mathrm{O}\left(R e^{2}\right),
\end{aligned}
$$

as $R e \rightarrow 0$. The stream function inside the liquid drop to first order in $R e$ is

$$
\begin{aligned}
\widehat{\psi}(r, \theta)= & -\frac{r^{2}\left(1-r^{2}\right) \sin ^{2} \theta}{4(1+\kappa)}\left[1+\frac{\kappa(2+3 \kappa)}{40(1+\kappa)^{2}} R e\right. \\
& \left.+\operatorname{Re} \frac{(2+3 \kappa)(5+4 \kappa)}{40(1+\kappa)^{2}}(1-r \cos \theta)\right]+\mathrm{O}(R e)^{2}
\end{aligned}
$$


as $R e \rightarrow 0$.

The stream functions (2.2) and (2.3) depend only on Re and $\kappa$ and are independent of the Weber number $W e$ and density ratio $\gamma$ where

$$
W e=\frac{\rho a U^{2}}{\sigma}, \quad \gamma=\frac{\hat{\rho}}{\rho}
$$

This is because the boundary conditions for the order Re solution are imposed on the zero order surface of the drop which is not deformed. The deformation of the drop to first order in $R e$ is proportional to $W e$ and depends on $\gamma$ and $\kappa$. The results therefore apply only for small Weber number.

\section{DETACHED WAKE}

We now investigate the properties of the wake behind the drop using the stream function (2.2).

\subsection{End points of the boundary of the standing eddy}

From $(2.2), \psi(r, \theta)=0$ on the surface of the drop $r=1$, along the axis of symmetry $\theta=0$ and $\theta=\pi$, and also along the curve

$$
\cos \theta=\left(1+\frac{8(1+\kappa)}{(2+3 \kappa) R e}\right) \frac{5(1+\kappa)\left[2(1+\kappa) r^{2}-\kappa r-\kappa\right] r}{\left[10(1+\kappa)^{2} r^{3}-5 \kappa(1-\kappa) r^{2}-\kappa r-\kappa(6+5 \kappa)\right]}
$$

Equation (3.1) is the boundary of the standing eddy behind the drop. It generates a surface of revolution about the line $\theta=0$. The end points of the boundary are its points of intersection with the axis of symmetry $\theta=0$ and are obtained by putting $\cos \theta=1$ in (3.1). The points therefore satisfy the cubic equation

$$
P(r)=0
$$

where

$$
\begin{aligned}
P(r)= & r^{3}-\frac{\kappa}{2(1+\kappa)} r^{2}-\frac{\kappa}{2(1+\kappa)}\left[1+\frac{(2+3 \kappa)(4+5 \kappa)}{40(1+\kappa)^{2}} R e\right] r \\
& +\frac{\kappa(6+5 \kappa)(2+3 \kappa)}{80(1+\kappa)^{3}} R e .
\end{aligned}
$$

For a solid sphere, $\kappa=\infty$, and (3.3) reduces to

$$
(r-1)\left(r^{2}+\frac{1}{2} r-\frac{3}{16} R e\right)=0 .
$$


The roots of (3.4) are

$$
r=-\frac{1}{4}\left[1+(1+3 R e)^{1 / 2}\right], \quad r=1, \quad r=\frac{1}{4}\left[(1+3 R e)^{1 / 2}-1\right] .
$$

In the limit $\kappa=\infty,(3.2)$ has one negative root which is not physical and two positive roots. Since $r=1$ is one end point, the boundary of the standing eddy is attached to the solid sphere. For the eddy to lie in the flow field the third root in (3.5) must be greater than unity, that is $R e>8[1,2]$. In the limit $\kappa=0$, which describes an inviscid gas bubble, (3.2) reduces to $r^{3}=0$. Equation (3.2) then has three coincident roots at $r=0$ which is in agreement with the result that there is no standing eddy behind a spherical bubble because no vorticity is generated upstream on the surface of an inviscid bubble [11].

Consider now $0<\kappa<\infty$. General properties of the roots of (3.2) can be obtained from Descartes' rule of signs [12]. Since there are two changes of sign in the coefficients of $P(r)$ equation (3.2) cannot have more than two positive roots. Since $P(0)>0$ and $P(r) \rightarrow \infty$ as $r \rightarrow \infty$ there will be either two distinct positive roots, two coincident positive roots or no positive roots. Further, since $P(1)>0$, the two positive roots, when they exist, will either both be greater than unity or both between 0 and 1 . When they exist, the two positive roots greater than unity are the end points of the boundary of the standing eddy. There is one change of sign in the coefficients of $P(-r)$ and therefore (3.2) cannot have more than one negative root. Since $P(0)>0$ and $P(r) \rightarrow-\infty$ as $r \rightarrow-\infty$ there will be exactly one negative root. This negative root is not physical.

In order to transform (3.2) to the standard form of a cubic equation let [12]

$$
r=s+\frac{\kappa}{6(1+\kappa)} .
$$

Equation (3.2) becomes

$$
s^{3}+3 H s+G=0
$$

where

$$
\begin{aligned}
& H(\kappa, R e)=-\left[\frac{\kappa(6+7 \kappa)}{36(1+\kappa)^{2}}+\frac{\kappa(2+3 \kappa)(4+5 \kappa)}{240(1+\kappa)^{3}} R e\right] \\
& G(\kappa, R e)=-\frac{(9+10 \kappa) \kappa^{2}}{108(1+\kappa)^{3}}+\frac{\kappa(2+3 \kappa)\left(25 \kappa^{2}+62 \kappa+36\right)}{480(1+\kappa)^{4}} R e .
\end{aligned}
$$

The standing eddy exists when all three roots of (3.7) are real, that is when [12]

$$
G^{2}+4 H^{3} \leq 0
$$

For condition (3.10) to apply it is necessary that $H<0$ which is satisfied by (3.8). When (3.10) holds, the solution of (3.7) is [12] 


$$
S_{n}=2(-H)^{1 / 2} \cos \left(\phi+\frac{2 n \pi}{3}\right), \quad n=0,1,2,
$$

where

$$
\cos 3 \phi=-\frac{G}{2(-H)^{3 / 2}}, \quad 0 \leq \phi \leq \frac{\pi}{3}
$$

Hence, transforming back from $s$ to $r$ using (3.6), the three real roots of (3.2) when they exist are

$$
r_{n}=\frac{\kappa}{6(1+\kappa)}+2\left[\frac{\kappa(6+7 \kappa)}{36(1+\kappa)^{2}}+\frac{\kappa(2+3 \kappa)(4+5 \kappa)}{240(1+\kappa)^{3}} R e\right]^{1 / 2} \cos \left(\phi+\frac{2 n \pi}{3}\right)
$$

where $n=0,1,2$ and $\phi$ is the solution of (3.12) in the range $0 \leq \phi \leq \pi / 3$.

Consider now the special case in which the two positive real roots are coincident. Coincident roots occur when the standing eddy first appears in the downstream wake. Equation (3.7) has three real roots with two roots the same and one different if $[12]$

$$
G^{2}+4 H^{3}=0
$$

and $H \neq 0$ and $G \neq 0$. Thus for two coincident real roots

$$
G= \pm 2(-H)^{3 / 2}
$$

and

$$
\cos 3 \phi=\mp 1, \quad 0 \leq \phi \leq \frac{\pi}{3} .
$$

If $\cos 3 \phi=+1$ then $\phi=0$ and it can be verified that $r_{1}=r_{2}<0$. To obtain the coincident positive roots we therefore consider $\cos 3 \phi=-1$. When $\cos 3 \phi=-1$, $\phi=\pi / 3$. Equation (3.13) with $n=1$ gives $r_{1}<0$. Equation (3.13) with $n=0$ and $n=2$ gives the two coincident positive roots, $r_{0}$ and $r_{2}$, Let $r_{A}=r_{0}=r_{2}$. Then

$$
r_{A}=\frac{\kappa}{6(1+\kappa)}+\left[\frac{\kappa(6+7 \kappa)}{36(1+\kappa)^{2}}+\frac{\kappa(2+3 \kappa)(4+5 \kappa)}{240(1+\kappa)^{3}} R e_{A}\right]^{1 / 2} 3 .
$$

The Reynolds number $R e_{A}$ satisfies (3.14) and has still to be determined. It is the Reynolds number at which the standing eddy first appears as $R e$ is increased from 
zero. The distance from the centre of mass of the drop to the point on the axis of symmetry where the eddy first appears is $r_{A}$.

The points of intersection of the boundary of the standing eddy with the axis of symmetry are $r_{0}$ and $r_{2}$ given by (3.13) for $n=0$ and $n=2$. The solution $r_{1}$ for $n=1$ yields the negative root. Graphs of $r_{0}$ and $r_{2}$ plotted against $R e$ for a range of values of $\kappa$ are shown in Figure 1. For $\kappa=\infty, r_{2}=1$ and the boundary of the standing eddy is attached to the surface of the solid sphere. For finite $\kappa, r_{2}>1$ and the boundary of the standing eddy is detached as shown for $\kappa=2,3,5,10$ and 30 . For finite $\kappa$ the wake is detached from the surface of the drop in agreement with numerical predictions [10]. When $R e=R e_{A}$ for given $\kappa$, then $r_{0}=r_{2}=r_{A}$. As $R e$ increases from $R e_{A}$ the length of the wake increases. The end point $r_{0}$ moves downstream while the end point $r_{2}$ moves upstream towards the surface of the drop.

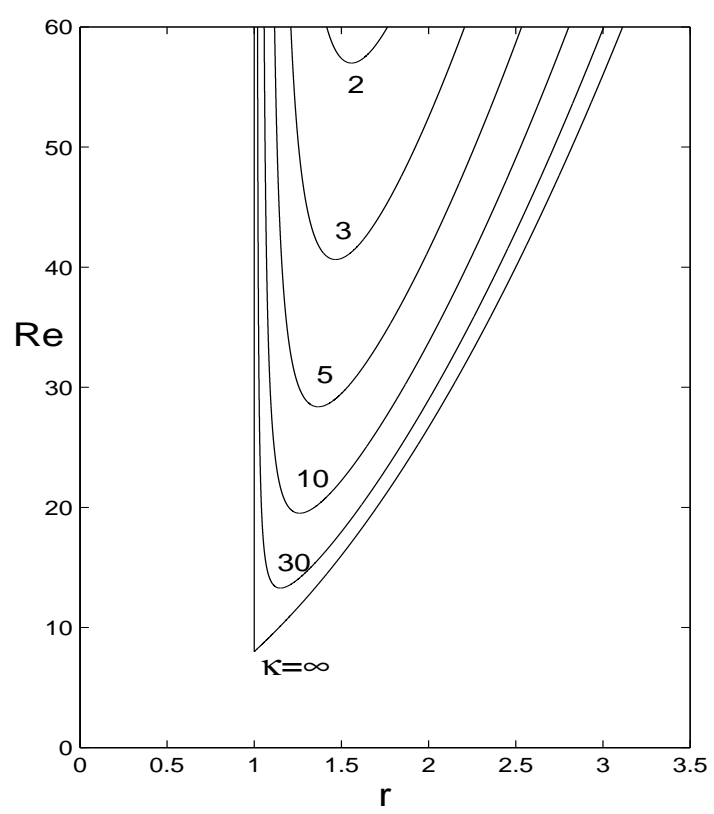

Figure 1: Points of intersection of the boundary of the standing eddy with the axis of symmetry plotted against Re for $\kappa=2,3,5,10,30$ and $\infty$.

The point on the axis of symmetry where the eddy first appears will be outside the drop if $r_{A}>1$. It follows from (3.17) that $r_{A}>1$ if $R e_{A}>R e^{*}$ where

$$
R e^{*}=\frac{120(2+3 \kappa)(1+\kappa)^{2}}{\kappa(2+3 \kappa)(4+5 \kappa)} .
$$

It is readily verified that for $0<\kappa<\infty, R e^{*}>8$ and that

$$
R e^{*}=8\left[1+\frac{38}{15 \kappa}+\frac{169}{225 \kappa^{2}}+\mathrm{O}\left(\frac{1}{\kappa^{3}}\right)\right]
$$


as $\kappa \rightarrow \infty$. In the limit of a solid sphere, $\kappa=\infty$ and $R e^{*}=8$. For a solid sphere $r_{A}=1$ and $R e^{*}=R e_{A}=8$ which is the prediction of Proudman and Pearson [2]. For a drop, $R e^{*} \neq R e_{A}$ because $r_{A}>1$ which is clearly seen in Figure 1 . In the next subsection we will investigate $R e_{A}$ and $r_{A}$.

Taylor and Acrivos [5] have observed that in all cases of physical significance the drop will be deformed into an oblate (flattened at $\theta=0$ and $\theta=\pi$ ) rather than a prolate (flattened at $\theta=\pi / 2$ ) spheroid. Thus $r_{A}>1$ will be exterior to the deformed drop in all cases of physical significance even when the deformation is included.

\subsection{Reynolds number for which standing eddy first appears}

The Reynolds number $R e_{A}$ for which the standing eddy first appears satisfies equation (3.14). Substituting (3.8) and (3.9) into (3.14) gives for $R e_{A}$ the cubic equation

$$
F(R e)=0
$$

where

$$
\begin{aligned}
F(R e)= & R e^{3}-\frac{5(1+\kappa)\left(1175 \kappa^{4}+7580 \kappa^{3}+15524 \kappa^{2}+13008 \kappa+3888\right)}{\kappa(2+3 \kappa)(4+5 \kappa)^{3}} R e^{2} \\
& +\frac{400(1+\kappa)^{3}\left(165 \kappa^{2}+322 \kappa+156\right)}{(2+3 \kappa)^{2}(4+5 \kappa)^{3}} R e+\frac{8000(1+\kappa)^{5}(8+9 \kappa)}{(2+3 \kappa)^{3}(4+5 \kappa)^{3}} .
\end{aligned}
$$

For solid sphere, $\kappa=\infty$ and (3.21) reduces to

$$
(R e-8)^{2}\left(R e+\frac{1}{3}\right)=0 .
$$

Thus when $\kappa=\infty,(3.20)$ has two coincident roots at $R e=8$ and one negative root, $R e=-1 / 3$ which is not physical. Hence for a solid sphere, $R e_{A}=8$ in agreement with the result derived from (3.5).

Consider now $0<\kappa<\infty$. There are two changes of sign in the coefficients of $F(R e)$ and therefore, by Descartes' rule of signs, $F(R e)=0$ cannot have more than two positive roots. But $F(\infty)=+\infty, F\left(R e^{*}\right)<0, F(8)<0$ and $F(0)>0$ where $R e^{*}$ is given by (3.18). Thus $F(R e)=0$ has at least two positive roots and hence there are exactly two positive roots, $R e_{S}$ and $R e_{L}$, where $R e_{S}<R e_{L}$. Then

$$
0<R e_{S}<8<R e^{*}<R e_{L} .
$$

Since $R e_{L}>R e^{*}, r_{A}\left(R e_{L}\right)>1$ where $r_{A}$ is given by (3.17) and therefore $R e_{L}=R e_{A}$, the value of the Reynolds number at which the standing eddy first appears behind the drop. Since $R e_{S}<R e^{*}, r_{A}\left(R e_{S}\right)<1$ and therefore $r_{A}$ will lie inside the drop 
which is not physical. There is one change of sign in the coefficients of $F(-R e)$ and therefore $F(R e)=0$ cannot have more than one negative root. Since $F(0)>0$ and $F(-\infty)=-\infty, F(R e)=0$ has

at least one negative root and hence exactly one negative root. This negative root is not physical.

In order to evaluate (3.17) analytically we will derive a perturbation solution for $R e_{A}$ for large values of $\kappa$. The perturbation solution is in a more suitable form to interpret the results than the exact solution. Let $\varepsilon=1 / \kappa$ and $y=R e$. Then(3.20) becomes

$$
\begin{aligned}
y^{3}-\frac{47}{3}\left(1+\frac{3091}{705} \varepsilon+\mathrm{O}\left(\varepsilon^{2}\right)\right) y^{2} & +\frac{176}{3}\left(1+\frac{67}{55} \varepsilon+\mathrm{O}\left(\varepsilon^{2}\right)\right) y \\
& +\frac{64}{3}\left(1+\frac{67}{45} \varepsilon+\mathrm{O}\left(\varepsilon^{2}\right)\right)=0,
\end{aligned}
$$

as $\varepsilon \rightarrow 0$. Consider first the straightforward perturbation expansion

$$
y=y_{0}+\varepsilon y_{1}+\mathrm{O}\left(\varepsilon^{2}\right) .
$$

Equation (3.24) becomes

$$
\begin{aligned}
& \left(y_{0}-8\right)^{2}\left(y_{0}+\frac{1}{3}\right) \\
& +\frac{\varepsilon}{3}\left[\left(9 y_{0}-22\right)\left(y_{0}-8\right) y_{1}-\frac{1}{45}\left(9273 y_{0}^{2}-9648 y_{0}-4288\right)\right]+\mathrm{O}\left(\varepsilon^{2}\right)=0 .
\end{aligned}
$$

Equate the coefficients of like powers of $\varepsilon$ in (3.26):

$$
\begin{aligned}
& \varepsilon^{0}: \quad\left(y_{0}-8\right)^{2}\left(y_{0}+\frac{1}{3}\right)=0, \\
& \varepsilon: \quad y_{1}=\frac{9273 y_{0}^{2}-9648 y_{0}-4288}{45\left(9 y_{0}-22\right)\left(y_{0}-8\right)} .
\end{aligned}
$$

From (3.27) $y_{0}=8,8$ and $-1 / 3$. When $y_{0}=-1 / 3$, it follows from (3.28) that $y_{1}=-1 / 225$ and therefore

$$
y=-\frac{1}{3}\left[1+\frac{\varepsilon}{75}+\mathrm{O}\left(\varepsilon^{2}\right)\right]
$$

as $\varepsilon \rightarrow 0$. When $y_{0}=8$, it follows from (3.28) that $y_{1}=\infty$. The assumed form (3.25) for the expansion of $y$ when $y_{0}=8$ is therefore not correct. 
To determine a valid expansion when $y_{0}=8$ consider $[13]$

$$
y=8+\varepsilon^{\nu} y_{1}+\varepsilon^{2 \nu} y_{2}+\mathrm{O}\left(\varepsilon^{3 \nu}\right),
$$

as $\varepsilon \rightarrow 0$ where $\nu>0$. Substituting (3.30) into (3.24) gives

$$
\frac{25}{3} y_{1}^{2} \varepsilon^{2 \nu}-\frac{102400}{27} \varepsilon+\mathrm{O}\left(\varepsilon^{1+\nu}\right)+\mathrm{O}\left(\varepsilon^{3 \nu}\right)=0 .
$$

Balancing the dominant terms, which are of order $\varepsilon^{2 \nu}$ and order $\varepsilon$, gives $\nu=1 / 2$. We therefore consider the perturbation expansion

$$
y=8+\varepsilon^{1 / 2} y_{1}+\varepsilon y_{2}+\varepsilon^{3 / 2} y_{3}+\mathrm{O}\left(\varepsilon^{2}\right),
$$

as $\varepsilon \rightarrow 0$. If (3.32) is substituted into (3.24), then (3.24) becomes

$$
\left(\frac{25}{3} y_{1}^{2}-\frac{102400}{27}\right) \varepsilon+y_{1}\left(\frac{50}{3} y_{2}-\frac{9248}{9}+y_{1}^{2}\right) \varepsilon^{3 / 2}+\mathrm{O}\left(\varepsilon^{2}\right)=0
$$

and equating the coefficients of like powers of $\varepsilon$ yields

$$
\begin{aligned}
& \varepsilon: \quad y_{1}= \pm \frac{64}{3} \\
& \varepsilon^{3 / 2}: \quad y_{2}=\frac{3}{50}\left(\frac{9248}{9}-y_{1}^{2}\right)=\frac{2576}{75} .
\end{aligned}
$$

Hence from (3.30),

$$
y=8\left[1 \pm \frac{8}{3} \varepsilon^{1 / 2}+\frac{322}{75} \varepsilon+\mathrm{O}\left(\varepsilon^{3 / 2}\right)\right]
$$

as $\varepsilon \rightarrow 0$. Since $\varepsilon=1 / \kappa$ and $y=R e$ the three roots (3.29) and (3.36) of the cubic equation (3.20) are

$$
\begin{aligned}
& R e=-\frac{1}{3}\left[1+\frac{1}{75 \kappa}+\mathrm{O}\left(\frac{1}{\kappa^{2}}\right)\right] \\
& R e_{S}=8\left[1-\frac{8}{3 \kappa^{1 / 2}}+\frac{322}{75 \kappa}+\mathrm{O}\left(\frac{1}{\kappa^{3 / 2}}\right)\right],
\end{aligned}
$$




$$
R e_{A}=8\left[1+\frac{8}{3 \kappa^{1 / 2}}+\frac{322}{75 \kappa}+\mathrm{O}\left(\frac{1}{\kappa^{3 / 2}}\right)\right],
$$

as $\varepsilon \rightarrow 0$. Only $R e_{A}$, given by (3.39), is physically significant.

In Figure 2 the numerical solution of (3.20) for $R e_{A}$ and the perturbation solution (3.39) are compared. The Reynolds number $R e^{*}$ given by (3.18) is also plotted. The perturbation expansion (3.39) is a good approximation for $5 \lesssim \kappa \leq \infty$. The numerical curve $R e=R e_{A}$ divides the $(\kappa, R e)$ plane into two regions. For $R e>R e_{A}$ the standing eddy exists downstream of the drop while for $R e<R e_{A}$ it does not exist. For $R e=R e_{A}$ the standing eddy first appears for the given value of $\kappa$.

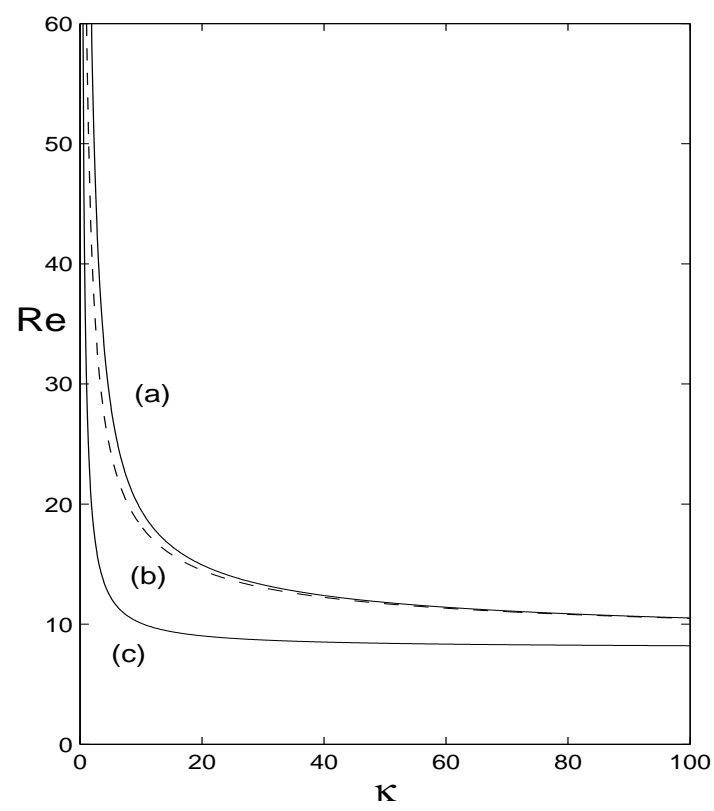

Figure 2: Graphs of (a) the numerical solution for $R e_{A}$, (b) the perturbation solution (3.39) for $R e_{A}$ and (c) $R e^{*}$ given by (3.18), plotted against $\kappa$. For a given value of $\kappa$, the standing eddy exists in the downstream wake if $R e>R e_{A}$.

The perturbation solution for $r_{A}$ is obtained by substituting (3.39) into (3.17) and expanding for large $\kappa$ :

$$
r_{A}=1+\frac{4}{5 \kappa^{1 / 2}}+\frac{18}{125 \kappa}+\mathrm{O}\left(\frac{1}{\kappa^{3 / 2}}\right)
$$

as $\kappa \rightarrow \infty$. The expansion (3.40) clearly shows that the standing eddy first appears at a point in the flow downstream of the drop. In Figure $3, r_{A}$ calculated 
from (3.17) using the numerical solution of (3.20) for $R e_{A}$ is compared with (3.40). The perturbation expansion underestimates the numerical solution but is a good approximation for $5 \lesssim \kappa \leq \infty$. The distance from the surface of the drop which we approximate as $r=1$, to where the standing eddy first appears, $r_{A}-1$, is approximately proportional to $\kappa^{-1 / 2}$ :

$$
r_{A}-1=\frac{4}{5 \kappa^{1 / 2}}\left(1+\frac{9}{50 \kappa^{1 / 2}}+\mathrm{O}\left(\frac{1}{\kappa}\right)\right)
$$

as $\kappa \rightarrow \infty$. As $\kappa$ increases the distance behind the drop to the point where the eddy first appears decreases and in the solid sphere limit, $\kappa=\infty$, the eddy may be imagined to penetrate through the surface and appear in the flow [1].

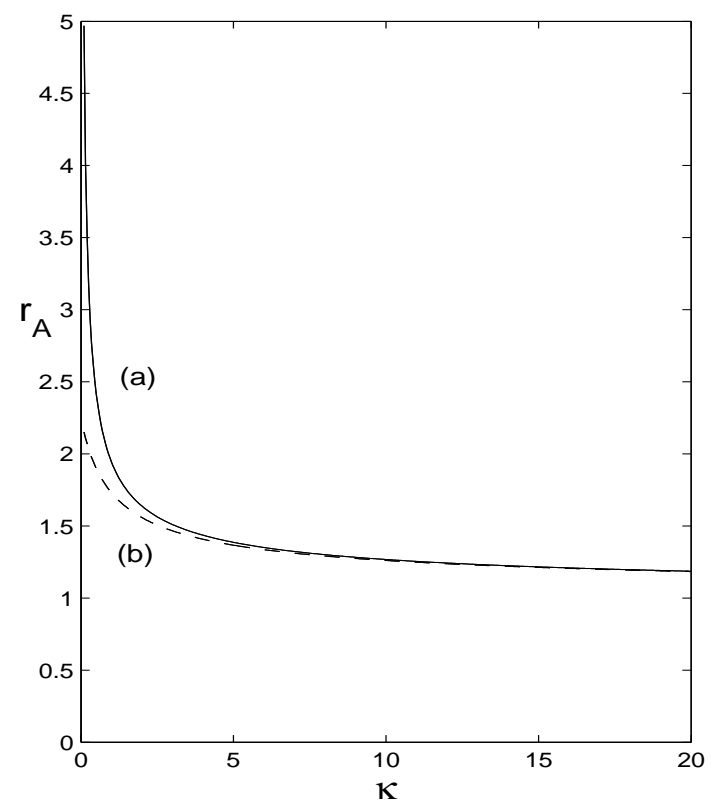

Figure 3: Distance from the centre of mass of the drop to the point where the standing eddy first appears, $r_{A}$, plotted against $\kappa$ : numerical solution (-), perturbation solution (3.40) (- - - ). 


\subsection{Stagnation points}

The radial and tangential components of the fluid velocity are

$$
v_{r}(r, \theta)=\frac{1}{r^{2} \sin \theta} \frac{\partial \psi}{\partial \theta}, \quad v_{\theta}(r, \theta)=-\frac{1}{r \sin \theta} \frac{\partial \psi}{\partial r} .
$$

Thus using (2.2) for $\psi(r, \theta)$,

$$
v_{r}(r, 0)=\frac{(r-1)}{r^{4}} P(r), \quad v_{\theta}(r, 0)=0,
$$

where $P(r)$ is defined by (3.3). Now the two distinct roots of $P(r)=0$ greater than unity are the end points of the boundary of the standing eddy on the axis of symmetry $\theta=0$. The end points of the boundary of the standing eddy are therefore stagnation points where $v_{r}=v_{\theta}=0$. The point at which the eddy first appears is also a stagnation point. At this point the two stagnation points are coincident and the radial velocity $v_{r}(r, 0)$ attains a local minimum value. The third stagnation point on the line $\theta=0$ is the rear stagnation point $r=1$. As $\kappa \rightarrow \infty$ the stagnation point closest to the drop as well as the point at which the eddy first appears tend to the rear stagnation point.

\section{STREAMLINES INSIDE THE LIQUID DROP}

The stream function inside the drop to first order in $R e$ is $(2.3)$. From $(2.3), \widehat{\psi}(r, \theta)=$ 0 on the surface of the drop $r=1$ and along the axis of symmetry, $\theta=0$ and $\theta=\pi$. When $0 \leq r<1$ and $\theta \neq 0$ and $\theta \neq \pi, \widehat{\psi}(r, \theta)<0$. There is therefore no boundary curve, $\widehat{\psi}(r, \theta)=0$, which divides the interior flow into two regions similar to the boundary of the standing eddy in the exterior flow. There is only one flow region in the axial plane inside the drop and the streamlines form closed curves which extend over the whole of the axial plane. This is in agreement with numerical solutions $[8,9,10]$.

In Figure 4 the streamlines inside and outside the drop are plotted for $\kappa=5$ and $R e=40$. The standing eddy exists downstream of the drop since from Figure $1, R e>R e_{A}$.

\section{RESULTS AND DISCUSSION}

The analysis predicts a detached wake for flow past a liquid drop, in contrast to the attached wake for flow past a solid sphere, which is consistent with numerical results and experiment. The existence of standing eddies downstream of the drop is due to the accumulation of vorticity generated upstream on the surface of the drop [10]. If $R e<R e_{A}$ convection will transport away the vorticity generated at the interface and no standing eddy will form. If $R e>R e_{A}$ then the vorticity generated at the interface will form a standing eddy behind the drop. The wake grows in size as $R e$ 


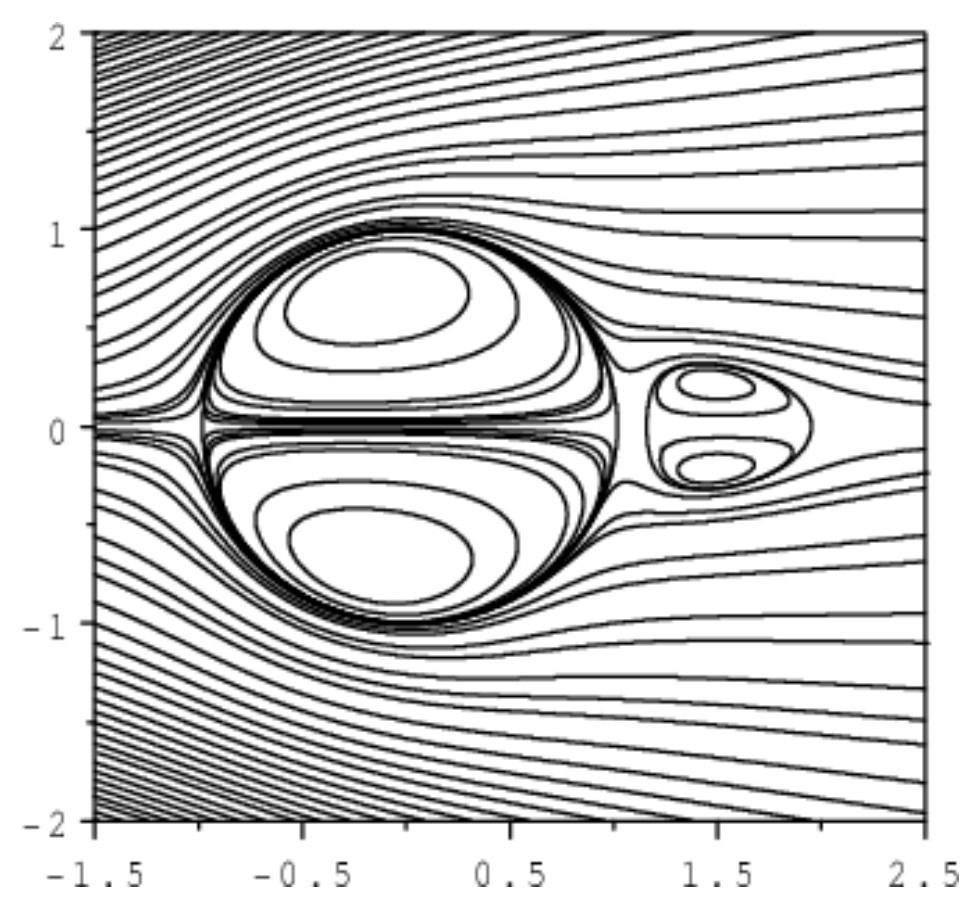

Figure 4: Streamlines for flow past a drop with $k=5$ and $R e=40$. The direction of the flow is from left to right.

increases and also as $\kappa$ increases as the no slip condition at the interface becomes a more effective source of vorticity. The wake is detached from the drop because of the internal flow of the drop. A liquid drop with an attached wake would require a secondary interior vortex [10]. As $\kappa$ increases the strength of the flow inside the drop decreases and the wake moves closer to the surface of the drop. In the limit $\kappa \rightarrow \infty$ there is no internal flow and the wake is attached.

The stream functions (2.2) and (2.3) to first order in $R e$ and therefore the results derived from them depend only on two parameters, $R e$ and $\kappa$, and apply only for small Weber number, $W e \ll 1$. The numerical and experimental results in the literature depend on four parameters, $R e, \kappa, W e$ and the density ratio $\gamma$. We will compare our analytical predictions with the numerical results of Dandy and Leal [10]. The Reynolds number and Weber number used by these authors is twice the value defined in (2.1) and (2.2).

Dandy and Leal [10] investigated the dependence of the structure of the wake on $W e$ for $R e=50$ and $\kappa=4$. For $W e=1$ the drop is almost spherical and $r_{0}$, the maximum extension of the wake downstream, is approximately 2.4 while the analytical prediction (3.13) is $r_{0}=2.14$. As $W e$ is increased the drop becomes an oblate spheroid and for $W e=2,3$ and $4, r_{0} \simeq 3,4.3$ and 4.6. The comparison between the analytical and numerical predictions is quite good for $W e=1$ but there is strong dependence on $W e$ and the analytical result is not reliable for $W e>1$.

The numerical plots of the streamlines in Dandy and Leal [10] can also be used to check the predictions of Figure 1. For $R e=50$ and $W e=2$ their streamline plots 
show that the standing eddy has not appeared for $\kappa=2$ but is present in the flow for $\kappa=4$ while for $R e=30$ the standing eddy has not appeared for $\kappa=4(W e=0.25)$ but is present for $\kappa=10(W e=2)$. These numerical results are consistent with Figure 1.

Equation(3.41) predicts that for $W e \ll 1$, the distance from the point where the eddy first appears to the surface of the drop decreases like $\kappa^{-1 / 2}$ as $\kappa \rightarrow \infty$. We were not able to find data to check this prediction.

The perturbation solution of Taylor and Acrivos was derived for $R e<1$ but it gave a good qualitative description of the flow features for $R e \lesssim 60$.

Van Dyke also found that the perturbation solution of Proudman and Pearson for $R e<1$ gave good predictions for the downstream end of the boundary of standing eddies for $R e \lesssim 60$. There are other examples in fluid mechanics where predictions have been made by giving the parameters values greater than permitted in the derivation of the solution. Longuet-Higgins [14] extended the range of the solution for capillary waves to predict the entrainment of air bubbles in wave troughs and Sostarecz and Belmonte [15] extended their model of a viscoelastic drop to predict that the boundary will self-intersect which could describe internal pinch-off at the trailing edge of the drop.

\section{CONCLUDING REMARKS}

Several new analytical results were presented. It was shown analytically that the wake is detached from the drop and that the end points of the boundary of the standing eddy on the axis of symmetry and the point at which the eddy first appears are stagnation points. We also saw analytically that inside the drop the streamlines from closed curves which extend over the whole of the axial plane. The end points of the boundary of the standing eddy were obtained as functions of the Reynolds number $R e$ and the viscosity ratio $\kappa$. Useful singular perturbation expansions in powers of $\kappa^{-1 / 2}$ were derived for the Reynolds number at which the downstream eddy first appears and for the point in the flow at which it first appears. It was predicted that the distance from the surface of the drop to the point where the eddy first appears is approximately proportional to $\kappa^{-1 / 2}$ for large $\kappa$.

Acknowledgement- This material is based upon work supported financially by the National Research Foundation, Pretoria, South Africa.

\section{REFERENCES}

[1] M. Van Dyke, Perturbation Methods in Fluid Mechanics, Academic Press, New York, 1964, chs 5 and 8.

[2] I.Proudman and J.R.A. Pearson, Expansion at small Reynolds numbers for the flow past a sphere and a circular cylinder, J. Fluid Mech. 2, 237-262,1957. 
[3] V.C. Jenson, Viscous flow round a sphere at low Reynolds numbers (<40), Proc. Roy. Soc. Lond. Ser. A, 249, 346-366, 1959.

[4] S. Taneda, Studies on wake vortices (III). Experimental investigation of the wake behind a sphere at low Reynolds numbers, Rep. Res. Inst. Appl. Mech. Kyushu Univ. 4, 99-105, 1956.

[5] T.D. Taylor and A. Acrivos, On the deformation and drag of a falling viscous drop at low Reynolds number, J. Fluid Mech. 18, 466-476, 1964.

[6] R.M. Wellek, A.K. Agrawal and A.H.P. Skelland, Shape of liquid drops moving in liquid media, AIChE Journal 12, 854-862, 1965.

[7] V.Y. Rivkind and G. Ryskin, Flow structure in motion of a spherical drop in a fluid medium at intermediate Reynolds numbers, Fluid Dyn.11, 5-12, 1976.

[8] D.L.R. Oliver and J.N. Chung, Steady flow inside and around a fluid sphere at low Reynolds numbers, J. Fluid Mech. 154, 215-230, 1985.

[9] D.L.R. Oliver and J.N. Chung, Flow about a fluid sphere at low to moderate Reynolds numbers, J. Fluid Mech. 177, 1-18, 1987.

[10] D.S Dandy and L.G. Leal, Buouancy-driven motion of a deformable drop through a quiescent liquid at intermediate Reynolds numbers, J. Fluid Mech. 208, 161-192, 1989.

[11] J. Magnaudet and I. Eames, The motion of high-Reynolds-number bubbles in inhomogeneous flows, Annual Rev. Fluid Mech. 32, 659-708, 2000.

[12] W. Briggs and G.H. Bryan, The Tutorial Algebra, University Tutorial Press, London, 1942, pp 448-450 and 496-508.

[13] A.H.Nayfeh, Introduction to Perturbation Techniques, John Wiley and Sons, New York, 1981, pp 39-43.

[14] M.S. Longuet-Higgins, Limiting forms of capillary-gravity waves, J. Fluid Mech. 194, 351-375, 1988.

[15] M.C. Sostarecz and A. Belmonte, Motion and shape of a viscoelastic drop falling through a viscous fluid, J. Fluid Mech. 497, 235-252, 2003. 